\title{
Performances of an Active Target GEM-Based TPC for the AMADEUS Experiment
}

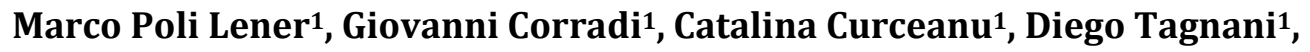 \\ Antonio Romero Vidal'2, Johann Zmeskal ${ }^{3}$ \\ ${ }^{1}$ Laboratori Nazionali di Frascati dell'INFN, Frascati, Italy \\ ${ }^{2}$ Universidad de Santiago de Compostela, Santiago de Compostela, Spain \\ ${ }^{3}$ Stefan Meyer Institute for Subatomic Physics, Vienna, Austria \\ Email: marco.polilener@Inf.infn.it
}

Received 7 April 2015; accepted 3 July 2015; published 6 July 2015

Copyright (C) 2015 by authors and Scientific Research Publishing Inc. This work is licensed under the Creative Commons Attribution International License (CC BY). http://creativecommons.org/licenses/by/4.0/

(c) $\underset{\mathrm{EY}}{0}$ Open Access

\begin{abstract}
In this paper, we present the $R \& D$ activity on a new GEM-based Time Projection Chamber (GEMTPC) detector for the inner region of the AMADEUS experiment, which is aiming to perform measurements of low-energy negative kaon interactions in nuclei at the DAФNE e+ e- collider at LNFINFN. A novel idea of using a GEM-TPC as a low mass target and detector at the same time comes motivated by the need of studying the low energy interactions of $\mathrm{K}$ - with nuclei in a complete way, tracking and identifying all of the produced particles. Even more, what makes the experimental proposal revolutionary is the possibility of using different gaseous targets without any other substantial intervention on the experimental setup, making it a flexible multipurpose device. This new detection technique applied to the nuclear physics requires the use of low-radiation length materials and very pure light gases such as Hydrogen, Deuterium, Helium-3, Helium-4, etc. In order to evaluate the GEM-TPC performances, a $10 \times 10 \mathrm{~cm}^{2}$ prototype with a drift gap of $15 \mathrm{~cm}$ has been realized. The detector was tested at the $\pi \mathrm{M} 1$ beam facility of the Paul Scherrer Institut (PSI) with low momentum pions and protons. Detection efficiency and spatial resolution, as a function of gas mixture, gas gain and ionazing particle, are reported and discussed.
\end{abstract}

\section{Keywords}

GEM, TPC, Tracking, Particle Identification

\section{Introduction}

An important, yet unsolved problem, in hadron physics is how the hadron masses and interactions change in the

How to cite this paper: Poli Lener, M., Corradi, G., Curceanu, C., Tagnani, D., Vidal, A.R. and Zmeskal, J. (2015) Performances of an Active Target GEM-Based TPC for the AMADEUS Experiment. Modern Instrumentation, 4, 32-41. 
nuclear medium. This topic could be investigated by means of "in-medium hadron-mass spectroscopy", producing bound states of a hadron by which to extract the hadron-nucleus potential and the in-medium hadron masses. The AMADEUS (Antikaon Matter At DAФNE Experiments with Unraveling Spectroscopy) experiment [1] [2] will study the low energy interactions of negative kaons with nucleons and nuclei. The dedicated AMADEUS setup will be implemented inside the KLOE [3] Drift Chamber (DC), in the free space between the beam pipe and the DC entrance wall. Currently, the main component of the experimental setup to be developed is an active target GEM-based [4] Time Projection Chamber [5] (GEM-TPC), which will act as low mass target and particles tracking detector at the same time.

A drawing of the dedicated AMADEUS setup surrounding the beam pipe within KLOE detector is given in Figure 1. The GEM-TPC will be $20 \mathrm{~cm}$ long with an inner diameter of $8 \mathrm{~cm}$ and an outer one of $40 \mathrm{~cm}$.

The main requirements can be summarized as:

- a spatial resolution better than $300 \mu \mathrm{m}$ in $\mathrm{X}-\mathrm{Y}$ and $500 \mu \mathrm{m}$ in $\mathrm{Z}$ with a magnetic field of $0.5 \mathrm{~T}$;

- a detector material budget lower than $0.5 \%$ of X0;

- a rate capability of $\sim 5 \mathrm{kHz} / \mathrm{cm}^{2}[6]$.

Since most of the above requirements are fulfilled by a GEM-TPC, the R \& D activity at the Laboratori Nazionali di Frascati (INFN) is mainly focused on the study of the detector performances with pure gas. A GEMTPC prototype of $10 \times 10 \mathrm{~cm}^{2}$ with a drift gap of $15 \mathrm{~cm}$ has been realized and tested both in laboratory and at the Paul Scherrer Institute (PSI). In Section 2 preliminary simulation studies on an active hydrogen target GEMTPC in the AMADEUS experiment are shown, while details of the prototype construction are described in Section 3. The detector performances obtained at the $\pi \mathrm{M} 1$ beam facility, in terms of detector efficiency and spatial resolution, are presented in Section 4.

\section{Simulation for a Hydrogen GEM-TPC}

Simulation studies have been performed with GEANT-4 [7] in order to investigate the use of an active target GEM-TPC in the AMADEUS experiment. The GEM-TPC is constrained to fit into the free volume between the DA $\Phi N E$ beam-pipe and the KLOE drift chamber. In the GEANT-4 simulation we set the following conditions:

- the phase space of $\Phi$ mesons is populated according to the recently proposed scheme of crab-waist collisions for DAФNE [8] [9];

- the charged particles undergo multiple scattering throughout the gas contained nside GEM-TPC, losing energy only by ionization;

- the GEM-TPC is filled with pure Hydrogen gas at standard condition $(300 \circ \mathrm{K}, 1 \mathrm{~atm})$;

- the particles move inside a magnetic field of $\mathrm{B}=0.5 \mathrm{~T}$.

As an example of this simulation study, we consider the $\mathrm{K}-\mathrm{p} \rightarrow \mathrm{K}-\mathrm{p}$ elastic scattering for a $127 \mathrm{MeV} / \mathrm{c}$ negative kaon, produced by the $\Phi$ decay, colliding with the gas target. The momentum of the scattered kaon ranges from $\sim 40 \mathrm{MeV} / \mathrm{c}$ to $127 \mathrm{MeV} / \mathrm{c}$ and the proton momentum can be as high as $\sim 165 \mathrm{MeV} / \mathrm{c}$ as shown in Figure 2: the kaons (blue line) with momentum larger than $100 \mathrm{MeV} / \mathrm{c}$ are emitted mainly at forward angles
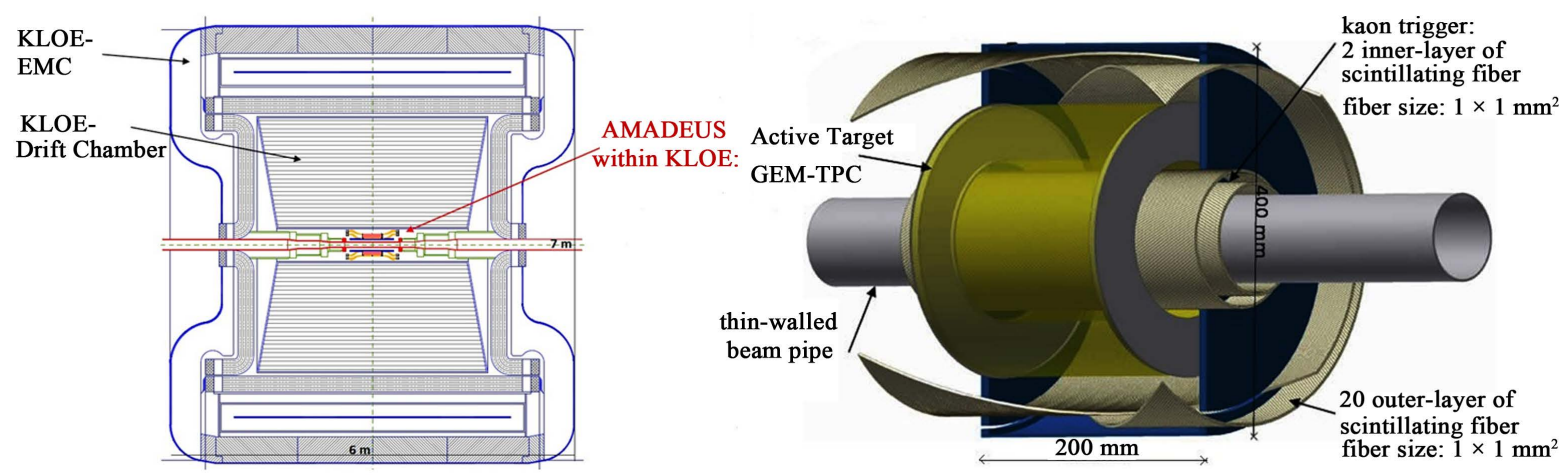

Figure 1. Cross-section of the KLOE detector including the AMADEUS inner setup inside the Drift Chamber (left) and the dedicated AMADEUS setup (right). From the beam pipe to the outer region: the Kaonic Trigger, the active target GEMTPC. 
along with protons (red line), otherwise for kaons with momentum lower than $100 \mathrm{MeV} / \mathrm{c}$, protons and kaons are emitted principally backward. Therefore the use of an active target TPC-GEM will allow tracking the considered elastic scattering in both forward and backward directions.

We also evaluated the effect of the multiple Coulomb scattering when a $127 \mathrm{MeV} / \mathrm{c}$ kaon crosses the Hydrogen gas target. As shown in Figure 3, the angular distribution is described for both the $\mathrm{x}$ - and y-coordinates (transverse plane to the beam direction) by a Gaussian distribution with a $\sigma \sim 85 \mu \mathrm{m}$, corresponding to a deflected kaon angle of less $1 \mathrm{mrad}$.

In Table 1 are reported for completeness the primary ionization of the hydrogen gas and the gas mixtures used at the PSI, which are estimated with the GARFIELD [10] simulation tool. Garfield is a computer program for the detailed simulation of two-and three-dimensional gas detectors and it is interfaced to the Magboltz [11] and HEED [12] programs for the computation of electron transport properties in nearly arbitrary gas mixtures and to simulate ionization of gas molecules by particles traversing the detector, respectively.

\section{The Amadeus GEM-TPC Prototype Production}

The GEM-TPC construction has been performed in a class 1000 clean room. The detector is composed by three GEM foils glued on Fiberglass (FR4) frames, defining the gaps between GEM themselves, then sandwiched

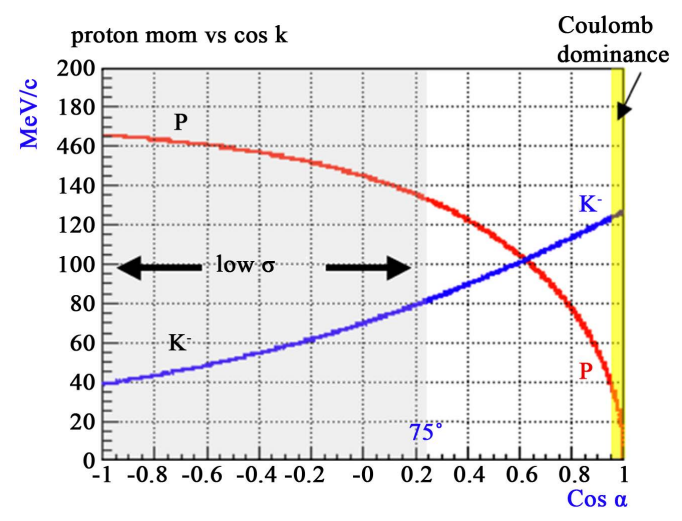

Figure 2. Kinematics of the $\mathrm{K}-\mathrm{p}$ elastic scattering: the blue curve shows the momentum of the emitted kaon versus the cosine of the angle between the incident kaon direction and the emitted one. The red curve shows the kinematics of the associated proton.
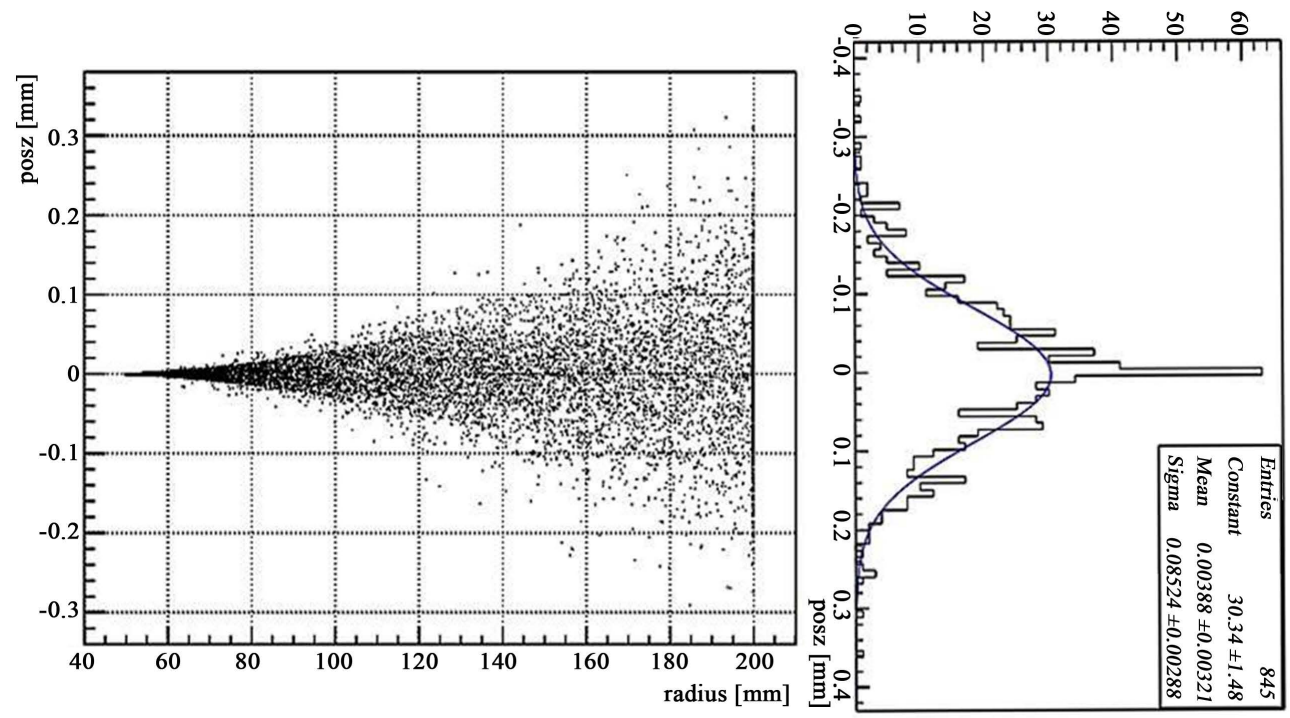

Figure 3. Multiple Coulomb scattering in the Hydrogen gas (left) and the relative angular distribution for an incident $127 \mathrm{MeV} / \mathrm{c}$ kaon (right). 
Table 1. Primary ionization clusters (mean value) and electrons per cluster (most probable value) for a pure hydrogen gas and the gas mixture tested at PSI for different particle momentum.

\begin{tabular}{|c|c|c|c|c|}
\hline \multirow[b]{2}{*}{ Gas Mixture } & & \multicolumn{2}{|c|}{ PSI } & \multirow{2}{*}{$\begin{array}{c}\text { DAFNE } \\
127 \mathrm{MeV} / \mathrm{c} \text { Kaon }\end{array}$} \\
\hline & & $170 \mathrm{MeV} / \mathrm{c}$ Pion & $440 \mathrm{MeV} / \mathrm{c}$ Proton & \\
\hline & $\mathbf{c l u} / \mathbf{c m}$ & & & $78.0 \pm 2.7$ \\
\hline \multicolumn{5}{|l|}{ Hydrogen 100} \\
\hline & $\mathrm{e}-/$ clu & & & $1.73 \pm 0.01$ \\
\hline & $\mathbf{c l u} / \mathbf{c m}$ & $4.0 \pm 0.7$ & $12.1 \pm 1.1$ & $31.1 \pm 1.8$ \\
\hline \multicolumn{5}{|l|}{ Helium 100} \\
\hline & e-/clu & $2.05 \pm 0.02$ & $2.03 \pm 0.01$ & $2.21 \pm 0.01$ \\
\hline & $\mathbf{c l u} / \mathbf{c m}$ & $40.2 \pm 2.0$ & $122.2 \pm 3.4$ & \\
\hline \multicolumn{5}{|c|}{ Ar/i-C4 H10 90/10 } \\
\hline & $e^{-}-$clu & $2.05 \pm 0.01$ & $2.04 \pm 0.01$ & \\
\hline
\end{tabular}

between a cathode and anode PCBs (Figure 4). The electric field uniformity in the drift volume is provided by a cylindrical field cage, which consists of two sets of copper strips ( $2.5 \mathrm{~mm}$ wide) on both sides of an insulating kapton foil (100 $\mu \mathrm{m}$ thick), where the outer strips cover the gaps between the inner strips. The potential on each ring is defined by a precision resistor chain located outside the gas volume. The cylindrical shape has been obtained by exploiting the vacuum bag technique [13] and rolling the copper-kapton foil onto machined polytetrafluorethylene (PTFE) cylinder that acts as mold. In this R \& D phase, the prototype has been encapsulated inside a gas tight box, which has been equipped by two $14 \times 12 \mathrm{~cm}^{2}$ windows covered by $10 \mu \mathrm{m}$ Mylar film, in order to reduce as much as possible the radiation length for a particle crossing the detector.

The detector gas tightness results to be lower than 2 mbar per day, corresponding to less than $100 \mathrm{ppmV}$ of residual humidity with a gas flux of $100 \mathrm{cc} / \mathrm{min}$.

\section{GEM-TPC Measurements and Performances}

\subsection{Gas Gain Measurement}

The effective gain of a triple-GEM detector has been measured for different gas mixtures and pure helium using a high intensity $5.9 \mathrm{keV}$ X-ray tube. Gain value is obtained from the ratio of the pad current (with high voltage on the GEM foils) to the current on the first GEM (with no high voltage on the GEM foils). As shown in Figure 5 , operational instability has been observed only for the pure helium gas at a gain greater than $\sim 3 \times 10^{4}$ due to the absence of a quencher in the gas.

\subsection{GEM-TPC Performances at the PSI Beam Facility}

The performances of the GEM-TPC prototype have been studied at the $\pi \mathrm{M} 1$ beam facility of the Paul Scherrer Institut (PSI) without magnetic field. The $\pi \mathrm{M} 1$ beam is a quasi-continuous high-intensity secondary beam providing pions or protons. The study of the detection efficiency and spatial resolution has been performed with a beam rate of $\sim 200 \mathrm{~Hz}$.

The prototype readout is composed by 4 columns of 32 pads of $3 \times 3 \mathrm{~mm}^{2}$ each. Each pad is connected to a front-end board based on CARIOCA-GEM chip [14] with a discriminator threshold of $\sim 2$ fC. Six MDT layers have been used in the test as an external track reference. The spatial resolution reached by the external tracker was $\sim 100 \mu \mathrm{m}$. The trigger of the setup has been generated by the coincidence of three scintillators $\mathrm{S} 1 \otimes \mathrm{S} 3 \otimes \mathrm{S} 2$, centred on the beam axis and covering an area of $\sim 12 \times 20 \mathrm{~mm}^{2}$ (Figure 6). Moreover, another scintillator, about $5 \mathrm{~m}$ far from the S1, S2, S3 cross, has been acquired in order to perform a measurement of the particle momentum crossing the prototype by means of time of flight. The measured momentum resolution for pion and proton beams in the momentum range between 100 and $440 \mathrm{MeV} / \mathrm{c}$ was less then $1 \%$. A schematic view of the 


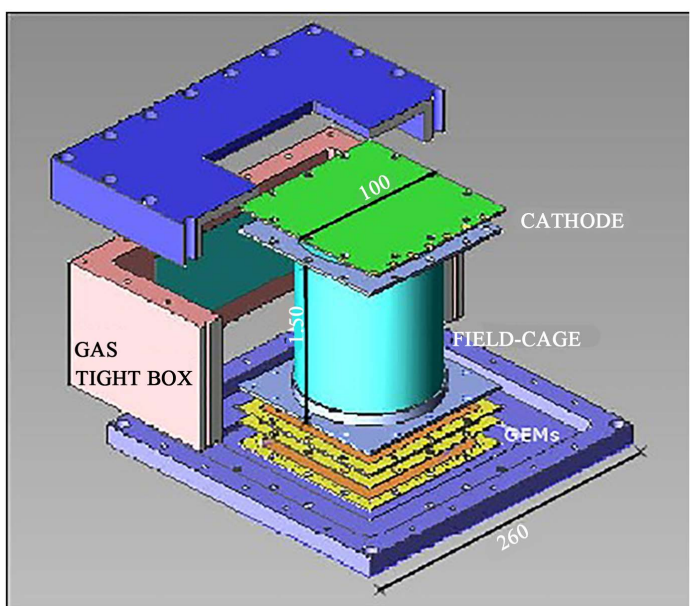

Figure 4. Exploded view of the GEM-TPC prototype.

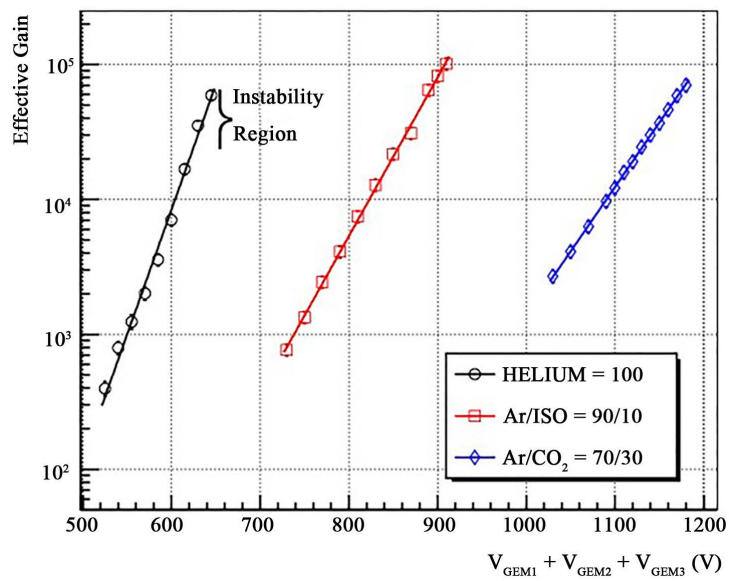

Figure 5. Effective gain as a function of the sum of the voltages applied on the three GEM foils for different gas mixtures and pure helium gas. The curve of the $\mathrm{Ar} / \mathrm{CO}_{2}=$ $70 / 30$ gas mixture is reported for comparison.

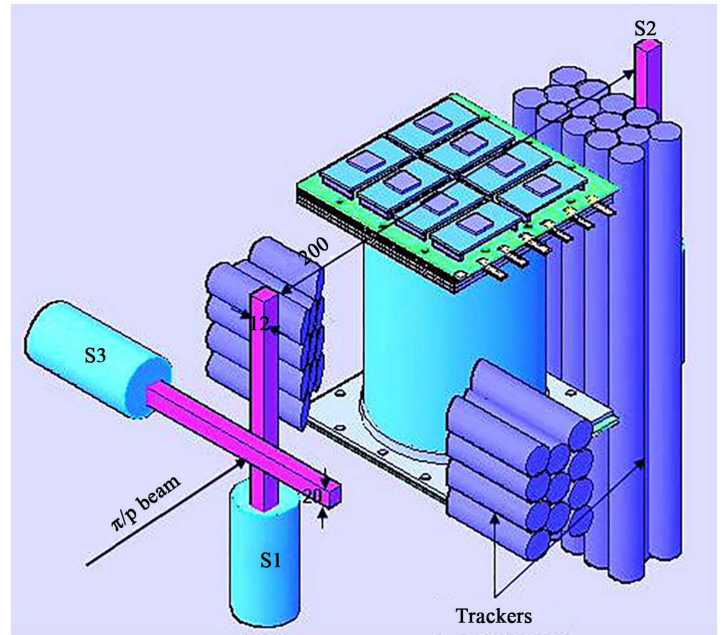

Figure 6. Schematic drawing of the experimental setup: the GEM-TPC prototype is located between the scintillators and the MDT layers used in the trigger and the external tracker, respectively. 
Data Acquisition (DAQ) used for the measurement of the GEM-TPC performances is shown in Figure 7.

In the following, unless otherwise stated, the beam is directed perpendicular to the drift axis of the GEM-TPC prototype and $4 \mathrm{~cm}$ far from the first GEM foil.

\subsubsection{Efficiency}

The single pad row detection efficiency has been evaluated considering the fraction of the hits in a single pad row with respect to a track selected by the external tracker.

An example of the single pad efficiency for the full detector and for each row is shown Figure 8. It is worth noticing that most of the pads have a detection efficiency larger than $98 \%$ except for the first 16 channels of the first row and some channels of the fourth row that are dead. A not full detection efficiency in the first and last pads of each row is clearly evident. The reason of this effect will be discussed more in detail in Section 4.2.3 and therefore the first and the last two pads of each row have been not considered in the following.

The detection efficiency, averaged on the 28 considered pads, as a function of the gain for $440 \mathrm{MeV} / \mathrm{c}$ proton and $170 \mathrm{MeV} / \mathrm{c}$ pion beams crossing the detector filled with $\mathrm{Ar} / \mathrm{i}-\mathrm{C} 4 \mathrm{H} 10$ (90/10) gas mixture and pure helium gas, is shown in Figure 9. As expected, due to the primary ionization of the particle in the gases used in the

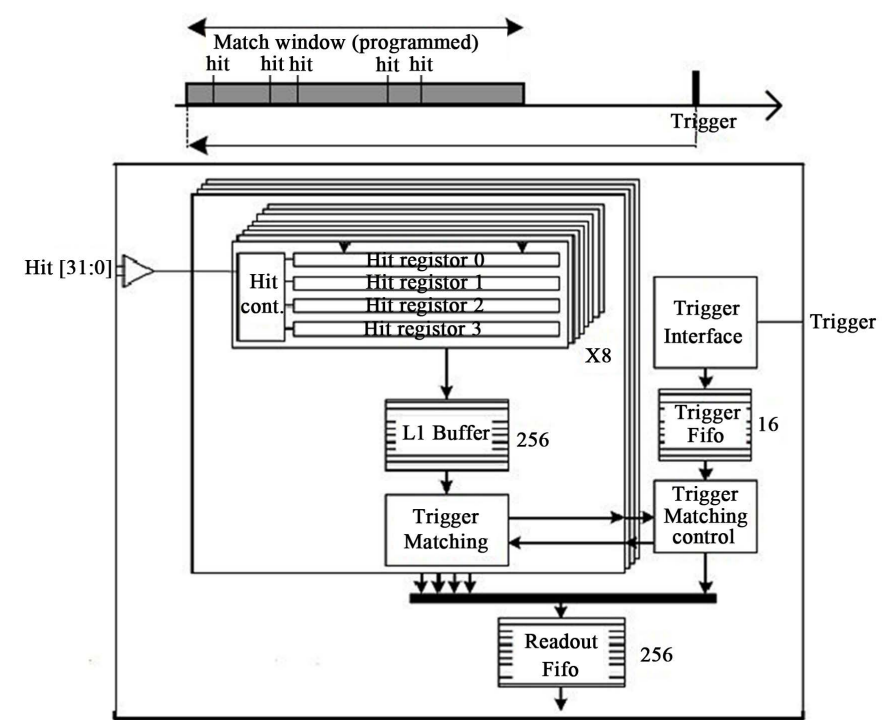

Figure 7. Sketch of the system used during the PSI beam test: hits storage occurred prior triggering, which has been properly delayed (top); the simplified Block Diagram of the Data Acquisition (DAQ) (bottom).
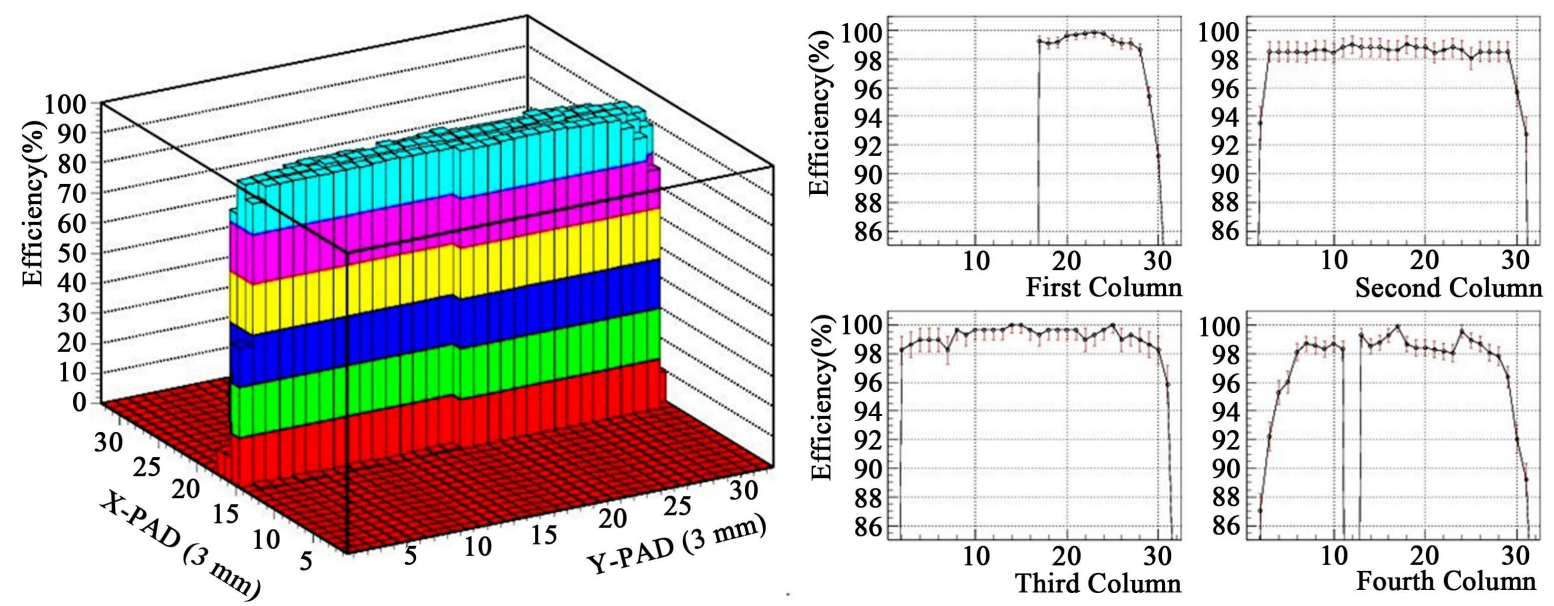

Figure 8. Single pad row efficiency for the full detector (left) and for each row (right) measured at the PSI beam facility. Each pad is $\sim 3 \times 3 \mathrm{~mm}^{2}$. 


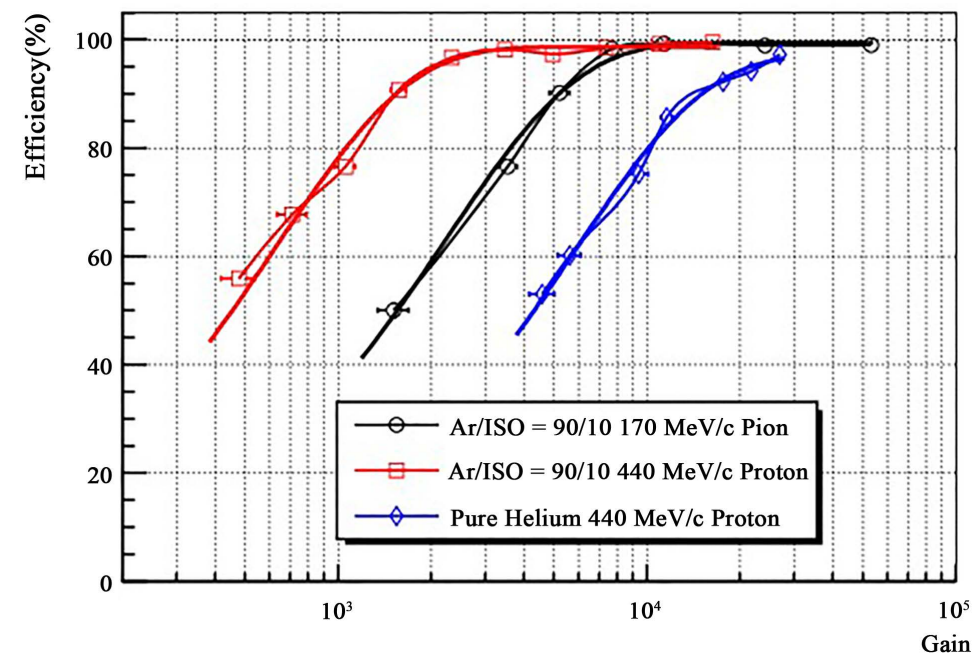

Figure 9. Detector efficiency as a function of the gain for $440 \mathrm{MeV} / \mathrm{c}$ proton and $170 \mathrm{MeV} / \mathrm{c}$ pion in $\mathrm{Ar} / \mathrm{i}-\mathrm{C} 4 \mathrm{H} 10$ gas mixture and pure helium measured at the PSI beam facility. The drift field is set to $0.4 \mathrm{kV} / \mathrm{cm}$ for all the measurements.

beam test, a full efficiency is reached with an isobutane-based gas mixture at lower gain, i.e. $\sim 3 \times 10^{4}$ for protons and $\sim 8 \times 10^{4}$ for pions, respectively, while for pure helium gas the maximum efficiency is $\sim 97 \%$ before to reach the instability region. The points in Figure 9 are fitted with a negative exponential function ${ }^{1}$ in order to evaluate the gain at which the detector efficiency is $97 \%, 95 \%$ and $90 \%$. These results have been plotted in Figure 10 as function of the primary ionization (simulated by GARFIELD) for $440 \mathrm{MeV} / \mathrm{c}$ proton and $170 \mathrm{MeV} / \mathrm{c}$ in Ar/i-C4 H10 (90/10) gas mixture and $440 \mathrm{MeV} / \mathrm{c}$ proton in pure helium gas (see Table 1). A function $\mathrm{y}=$ $\mathrm{p} 0 / \mathrm{x}^{\mathrm{p} 1}$ has been used to fit the points of Figure $10^{2}$ in order to estimate the gain, and accordingly the efficiency, for a $127 \mathrm{MeV} / \mathrm{c}$ kaon crossing the active target GEM-TPC filled with a pure helium or a pure hydrogen gases as foreseen in the AMADUES experiment. A suitable gain value of $\sim 8 \times 10^{3}$ or $4 \times 10^{3}$ for a helium or hydrogen gases (vertical dotted lines), respectively, will allow to reach a detection efficiency of $\sim 95 \%$ and to operate the detector in stable condition.

\subsubsection{Spatial Resolution}

The spatial resolution has been evaluated by the residuals between the hit position in a single pad row (excluding it from the fit) and the track defined by the six MDT layers. The spatial resolution is then calculated as an average of the 28 considered pads:

$$
\frac{1}{28} \sum_{i=1}^{28} \sqrt{\left(\sigma_{i-\text { residual }}^{2}-\sigma_{\text {externaltracker }}^{2}\right)}
$$

Figure 11 shows that the behavior of the spatial resolution depends on the gain and the ionizing particle: the higher is the gain and the greater is the primary ionization produced by the particle crossing the detector, the higher is the spatial resolution. Moreover, the different level of the spatial resolution reached by the prototype at high gain can be explained by the different transverse diffusion of the tested gas, which are $\sim 350 \mu \mathrm{m} / \mathrm{cm}$ and $\sim 600 \mu \mathrm{m} / \mathrm{cm}$ at $0.4 \mathrm{kV} / \mathrm{cm}$ (provided by GARFIELD) for the $\mathrm{Ar} / \mathrm{i}-\mathrm{C} 4 \mathrm{H} 10$ gas mixture and the pure helium gas, respectively.

\subsubsection{Edge Effect Due to the Field Cage}

Low and/or not full detection efficiency has been measured on the edge of each pad rows as clearly visible in Figure 8. Such effects are due to different reasons:

- the length of each pad row is $102 \mathrm{~mm}$ while the effective diameter of the cylindrical field-cage is $\sim 100 \mathrm{~mm}$.

${ }^{1} \mathrm{Eff}=1-\mathrm{e}-\mathrm{G}$, where Eff and $\mathrm{G}$ are the detector efficiency and gain, respectively.

${ }^{2} \mathrm{G}=$ ntot $/ \mathrm{nt}$, where ntot and $\mathrm{nt}$ are the number of secondary electrons inducing a signal on the readout and the number of primary electrons produced by a ionizing particle, respectively. 


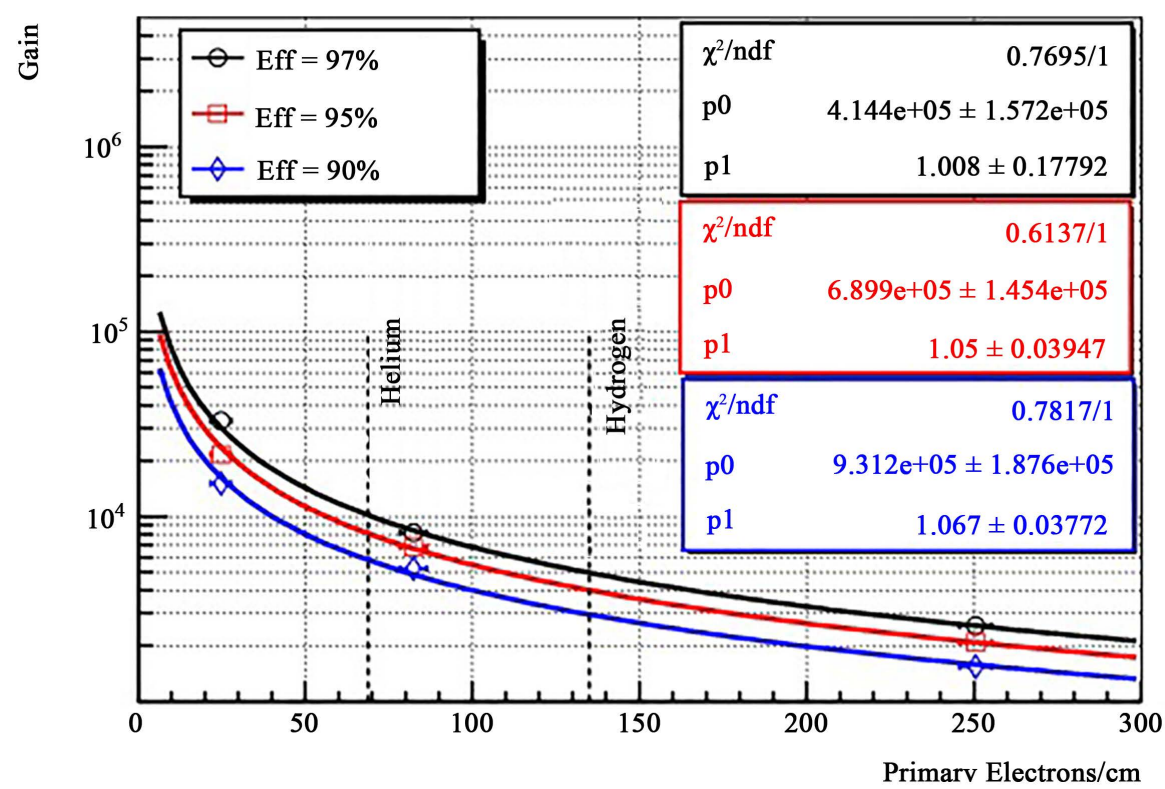

Figure 10. Gain (estimation from the previous fit) as a function of the primary electrons (simulated) for different value of the accepted detector efficiency measured at the PSI beam facility ( $97 \%$ black line; $95 \%$ red; $90 \%$ blue).

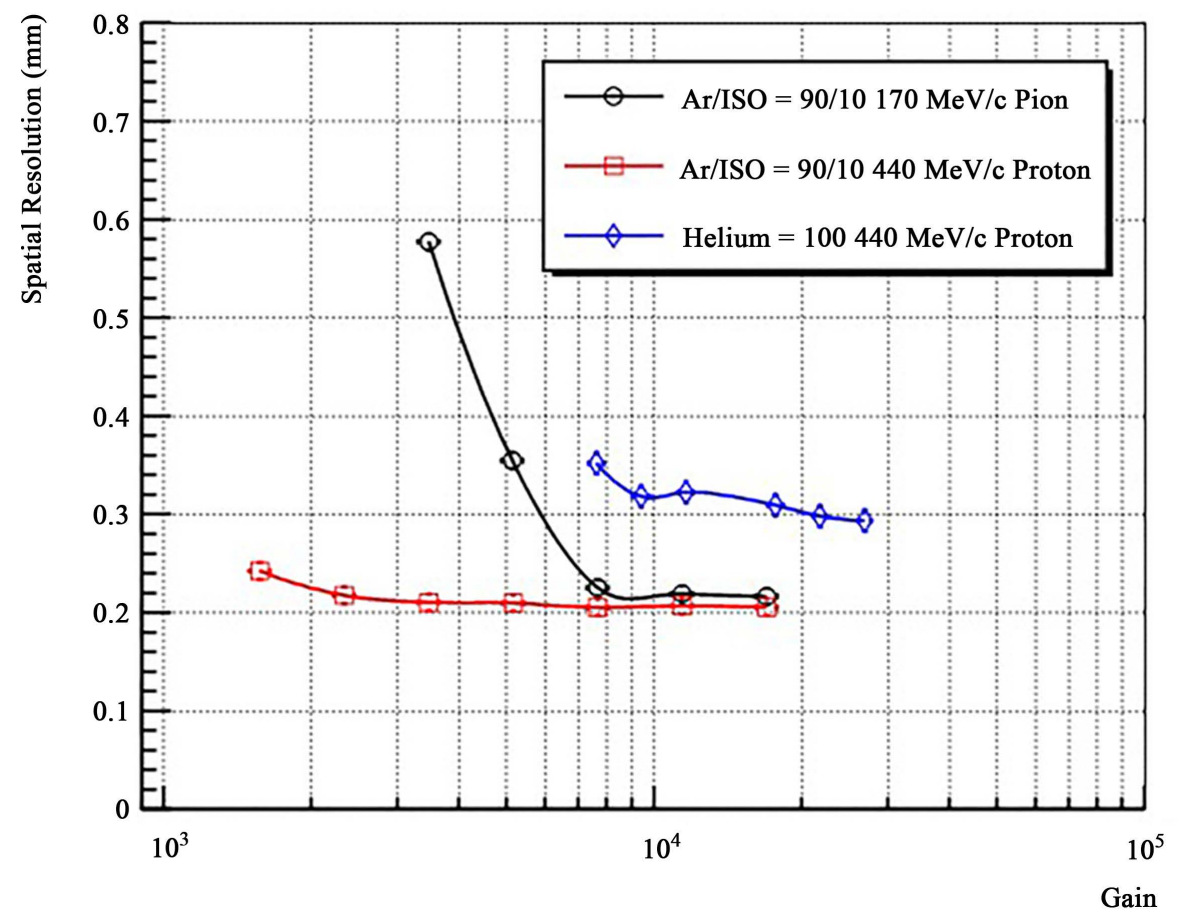

Figure 11. Spatial resolution as a function of the gas gain for proton of $440 \mathrm{MeV} / \mathrm{c}$ and pion of $170 \mathrm{MeV} / \mathrm{c}$ in Ar/i-C4 H10 gas mixture and pure helium measured at the PSI beam facility. The drift field is set to $0.4 \mathrm{kV} / \mathrm{cm}$ for all the measurements.

This means that the first and the last pad of each row collects about $2 / 3$ of the charge with respect to the other pads of the row;

- electric distortion of the field in the drift gap mainly near the field-cage are notcompletely cured;

- the primary electrons produced in the drift gas and drifting toward the first GEM can be collected by the internal strips of the field-cage (Figure 12). In any case all these effects are fully reduced drifting away from the field-cage by $\sim 5 \mathrm{~mm}$. 


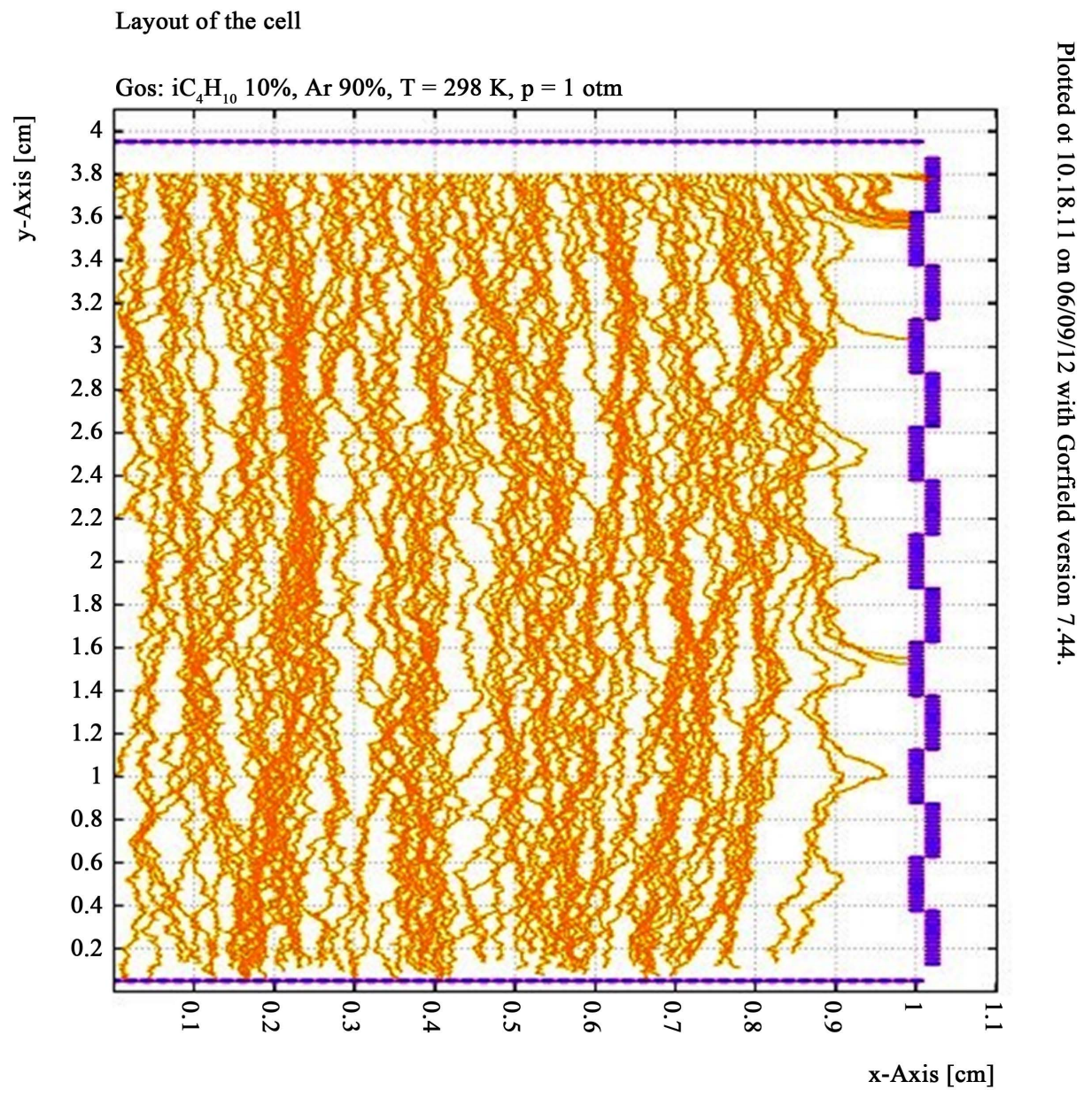

Figure 12. Garfield simulation of primary electrons motion in the drift gap.

\section{Conclusions}

The $\mathrm{R} \& \mathrm{D}$ activity on a new active target GEM-based TPC for the inner region of the AMADEUS setup has started at the Laboratori Nazionali di Frascati (INFN).

A GEM-TPC prototype with a drift gap of $15 \mathrm{~cm}$ has been successfully built and tested at the $\pi \mathrm{M} 1$ beam facility of the Paul Scherrer Institute with low momentum pion and proton beams.

The measurement of the detector performances, in terms of detection efficiency and spatial resolution, as a function of the gain and ionizing particle has been reported for an isobutane-based gas mixture and a pure helium gas. The detector performances have shown a detection efficiency of $99 \%$ and a spatial resolution of $\sim 200$ $\mu \mathrm{m}$ for $\mathrm{Ar} / \mathrm{i}-\mathrm{C} 4 \mathrm{H} 10=90 / 10$ gas mixture, while for a pure helium gas the detector performances have reached a $97 \%$ of efficiency and a $\sim 250 \mu \mathrm{m}$ of spatial resolution.

The achieved results could open the possibility to use such GEM-TPC filled with pure light gas as a low mass target and tracker detector at the same time in the AMADEUS environment.

\section{Acknowledgements}

The authors would like to thank the coordinator of the PSI beam lines, Dr. Konrad Deiters, for the excellent cooperation and support; M. Pistilli for his suggestions during the prototype design phase and for his highly qualified technical assistance during the construction.

Part of this work was supported by the European Community-Research Infrastructure Integrating Activity "Study of Strongly Interacting Matter" Hadron Physics 2 (HP2), Grant Agreement No. 227431, and HadronPhysics 3 (HP3), Contract No. 283286, under the Seventh Framework Programme of EU. 


\section{References}

[1] AMADEUS Letter of Intent. www.lnf.infn.it/lnfadmin/direzione/roadmap/LOI_MARCH_AMADEUS.pdf

[2] The AMADEUS Collaboration (2007) AMADEUS Phase-1: Setup and Roll-In Proposal, LNF-07/24(IR).

[3] Adinolfi, D., et al. (2001) The KLOE Drift Chamber. Nuclear Instruments and Methods in Physics Research Section A, 461, 25-28. http://dx.doi.org/10.1016/S0168-9002(00)01157-8

[4] Sauli, F. (1997) GEM: A New Concept for Electron Amplification in Gas Detectors. Nuclear Instruments and Methods in Physics Research Section A, 386, 531-534. http://dx.doi.org/10.1016/S0168-9002(96)01172-2

[5] Nygren, D. (1975) The Time Projection Chamber, SLAC and LBL Reports PEP-144 (1974), and PEP-198.

[6] Poli Lener, M. (2006) Triple-GEM Detectors for the Innermost Region of the Muon Apparatus at the LHCb Experiment. Ph.D. Thesis, Università degli Studi Roma Tor Vergata, Rome.

[7] Agostinelli, S., et al. (2003) GEANT4-A Simulation Toolkit. Nuclear Instruments and Methods in Physics Research Section A, 506, 250-303. http://dx.doi.org/10.1016/S0168-9002(03)01368-8

[8] Alesini, D., et al. (2006) DAФNE Upgrade for SIDDHARTA RUN, LNF-06/33(IR).

[9] Milardi, C. (2008) DAФNE Interaction Regions Upgrade, <arXiv:0803.1450v1>.

[10] Veenhof, R. (1998) Garfield, Recent Developments. Nuclear Instruments and Methods in Physics Research Section A, 419, 726-730. http://dx.doi.org/10.1016/S0168-9002(98)00851-1

[11] Biagi, S.F. (1999) Monte Carlo Simulation of Electron Drift and Diffusion in Counting Gases under the Influence of Electric and Magnetic Fields. Nuclear Instruments and Methods in Physics Research Section A, 412, 234-240. http://dx.doi.org/10.1016/S0168-9002(98)01233-9

[12] Smirnov, I.B. (2005) Modeling of Ionization Produced by Fast Charged Particles in Gases. Nuclear Instruments and Methods in Physics Research Section A, 554, 474-493. http://dx.doi.org/10.1016/j.nima.2005.08.064

[13] Bencivenni, G. et al. (2007) A Novel Idea for an Ultra-Light Cylindrical GEM Based Vertex Detector. Nuclear Instruments and Methods in Physics Research Section A, 572, 168-169. http://dx.doi.org/10.1016/j.nima.2006.10.173

[14] Poli Lener, M., et al. (2007) The Commissioning of the GEM Detector for the Muon Apparatus of the LHCb Experiment. IEEE Nuclear Science Symposium Conference Record, 6, 4671-4676. http://dx.doi.org/10.1109/NSSMIC.2007.4437149 